\title{
Hypnotics and anxiolytics
}

\author{
Brian R Ballinger
}

During the past 20 years benzodiazepines have been widely prescribed to treat anxiety states and sleep disorders. The problem of drug dependence, however, and discussion of alternatives including non-drug methods of management have led to increasing caution in their use. This article considers the role of drugs in treating, firstly, anxiety and then sleep disorders.

\section{Anxiolytics}

Anxiety is a normal reaction but when severe and disabling it becomes pathological. Phobias are distinguished from anxiety, and some classifications also separate panic attacks. Patients should be assessed carefully to detect associated problems in their lives and to seek evidence of underlying illness, such as depression and organic brain disease, that may present as anxiety. Conversely it should be remembered that some patients presenting with anxiety have an underlying physical illness. Drugs are often not necessary to treat neurotic anxiety, and in many instances a simple form of psychotherapy or sometimes behaviour therapy may succeed.

\section{BENZODIAZEPINES}

The benzodiazepines have anxiolytic and hypnotic effects, although some have been marketed mainly to treat anxiety. The original benzodiazepine, chlordiazepoxide, has now been available for more than 20 years and it has been followed by many others. 'Although not all these drugs are new, it seems appropriate to review their present role in treatment, as advantages such as relative safety in overdose have led to their widespread use. The recently introduced specific benzodiazepine antagonist, flumazenil, may be of use in some cases of overdose. Although the structure of a particular benzodiazepine molecule may influence its activity-for example the 1,5benzodiazepine, clobazam, may be relatively less sedative than the 1,4-benzodiazepines-in practice the duration of action mainly determines what benzodiazepine to use. The speed of absorption is also relevant, and the high lipid solubility of diazepam may make the effect of a single dose short lived.

\section{Duration of action}

Because many benzodiazepines are metabolised in the liver to produce further active forms that are eliminated from the body more slowly than the parent molecule, care has to be taken when assessing information on the duration of action of these drugs. For example, medazepam has an elimination half life of one to two hours but is metabolised to oxazepam, which has a half life of 6-24 hours. Pharmacodynamic studies that record the duration of measurable effects such as sedation are the best source of this information, but because such studies are difficult to undertake a full description is not always available. Prescribers should be alert to the possibility of cumulative hangover effects, especially in elderly people and those whose hepatic function may be impaired. Particular caution is needed with the drugs that have long elimination half lives and those that undergo appreciable oxidative metabolism in the liver (all except temazepam, lormetazepam, oxazepam, and lorazepam).

\section{Dependence}

Dependence on the benzodiazepines does occur and is now regarded as a serious problem,,$^{23}$ particularly with longer term treatment and in patients with some types of personality disorder. Patients taking these drugs, even in therapeutic doses, may develop a physical withdrawal syndrome. The main symptom of this is anxiety, which usually subsides in two to four weeks but sometimes lasts longer, although many patients would have been prone to anxiety before treatment was started. In addition, depression, nausea, depersonalisation, and perceptual changes such as intolerance of loud noises, bright lights, or touch may occur. Insomnia may also be expected but the symptoms are variable. Occasionally epileptic seizures, confusion, and visual hallucinations may occur.

Stopping treatment with short acting benzodiazepines leads to withdrawal symptoms within about two to three days, whereas with longer acting drugs there may be a delay of seven days. Patients being weaned from benzodiazepines need close supervision and support, and the drugs should be withdrawn slowly over weeks or even months. Sometimes $\beta$ blocking drugs and psychological aids such as relaxation therapy help withdrawal.

\section{Other effects}

Drowsiness may be a hazard, particularly when operating machinery or driving, and mental confusion may be precipitated or made worse, particularly in older people. Memory may be impaired and incoordination or ataxia may occur. Loss of control leading to aggressive behaviour or self poisoning is a problem in a few patients.

Age - The production of active metabolites of some drugs depends on oxidative metabolic pathways in the liver and the elimination of conjugated metabolites depends on renal function. Hepatic and renal functions are known to deteriorate with age and the elderly therefore eliminate benzodiazepines more slowly, which may result in higher peaks of concentration in the body and prolonged duration of action. The aging brain also appears to be more sensitive to the effects of these drugs. For these reasons benzodiazepines usually need to be given to elderly people in smaller doses, and the shorter acting drugs are generally indicated to avoid hangover effects when benzodiazepines are used as hypnotics.

Interactions-Alcohol and benzodiazepines taken concomitantly may result in greater impairment of psychomotor function than either agent alone. There are many possible explanations for this observation, but acute alcohol intake may inhibit the metabolism of most benzodiazepines, probably except those that do not undergo oxidation (see below). The usual effect of the combination of alcohol and benzodiazepines is an increase in the sedative effects of the benzodiazepine, but cases of aggressive behaviour have occurred.

Another commonly used drug that may theoretically cause considerable interaction is cimetidine, which 
inhibits oxidative metabolism in the liver. Only four available benzodiazepines (temazepam, lormetazepam, oxazepam, and lorazepam) are not metabolised by this pathway and would be free of this effect. Alternatively, ranitidine, which does not inhibit oxidative metabolism, should be substituted for cimetidine.

The decision to start giving benzodiazepines for anxiety should not be taken lightly, and treatment should normally be limited to patients whose anxiety is causing unacceptable distress. ${ }^{+5}$ Their use should be avoided for mild anxiety. Treatment should usually be for a limited period only because of the increasing risk of dependence developing, and the Committee on Safety of Medicines recommends treatment for two to four weeks only. Intermittent flexible dose treatment in which medication is taken occasionally when symptoms are severe, but never continuously, may have advantages. The shorter acting drugs such as oxazepam or lorazepam have sometimes been preferred for acute anxiety, although withdrawal symptoms may be more of a problem with these drugs.

Longer term treatment with benzodiazepines is controversial and many doctors use it for certain patients with severe neurosis. Given the potential problems of dependence and the present climate of opinion, however, this practice should usually be avoided except probably for a few patients already receiving long term treatment whose condition is stable and for whom withdrawal would cause serious problems.

In view of the similarities between different benzodiazepines, a limited list of drugs available for NHS prescription has been introduced. It includes diazepam, chlordiazepoxide, lorazepam, oxazepam, and some drugs marketed as hypnotics (nitrazepam, loprazolam, lormetazepam, temazepam, and triazolam), but changing patients from one drug to another has sometimes proved difficult in practice.

\section{BUSPIRONE}

Buspirone is a new drug that is now marketed as an anxiolytic, although hypnotic or anticonvulsant effects are not claimed for it. $^{6}$ It has an entirely different structure from other anxiolytics, and the recommended dose range is $15-30 \mathrm{mg}$ a day in divided doses. It is well absorbed but undergoes extensive first pass metabolism; it is highly protein bound and only $1 \%$ is excreted unchanged.

Trials have shown that buspirone improves symptoms of anxiety, though no more effectively than the benzodiazepines. It is less sedative than diazepam and does not relieve benzodiazepine withdrawal symptoms. Buspirone is not very effective in patients who have not responded to benzodiazepines, and its anxiolytic effect is relatively slow to develop, which may be a disadvantage in some circumstances.

Side effects are not usually severe, although gastrointestinal upset, dizziness, and headache have occurred. In contrast to treatment with benzodiazepines, no withdrawal symptoms have been reported on stopping treatment after six weeks to six months and there is no evidence of tolerance. Buspirone does not appear to have an additive effect with alcohol and has not been shown to impair cognitive function.

It is too early to assess the place of buspirone in treating anxiety as experience with this drug is limited. The lack of reports of drug dependence is encouraging for longer term treatment, but reports of dependence on other anxiolytics were slow to appear. The absence of sedative effects may be valuable for some patients.

\section{OTHER DRUGS}

Barbiturates can no longer be recommended as they are dangerous in overdose, readily cause psychological and physical dependence, and are potent inducers of liver enzymes. Meprobamate is less effective than the benzodiazepines and less safe. $\beta$ Receptor blockers such as propranolol have a role in the management of some patients as they control the somatic symptoms of anxiety, such as tremor and palpitations? They are sometimes useful for treating anxiety associated with public speaking and similar activities, but the type of patient who responds to $\beta$ blockers has not been defined fully.

Small doses of neuroleptics such as trifluoperazine have sometimes been used to treat anxiety, and they have the advantage of not causing dependence. The potential hazards of these agents, however, such as tardive dyskinesia, make them unsuitable for the long term treatment of neuroses. The sedative antidepressants have an anxiolytic effect and may be useful in managing anxiety when coexisting depressive symptoms justify antidepressant treatment. Tricyclic antidepressants, however, also seem to have a part to play in treating some patients who are liable to recurrent panic attacks and some other people with anxiety states. ${ }^{*}$ The antidepressant clomipramine is reported to be effective in some sufferers from obsessive compulsive disorder. The monoamine oxidase inhibitors such as phenelzine may be useful in managing some patients with agoraphobia, usually after behaviour therapy has been tried.

\section{Hypnotics}

Each patient's condition should be evaluated carefully before a hypnotic is prescribed. ${ }^{9}$ Not all people complaining of difficulties in sleeping really have insomnia, and sleep patterns and requirements vary with age and among people. Particular causes of insomnia should be sought, including the use of stimulant drinks such as tea or coffee at bedtime, discomfort, and noise. Painful physical illness or depression may be associated with sleep problems and these may resolve with appropriate treatment. As with anxiety, psychological methods of treatment have a role in managing insomnia.

Hypnotics should not be given unless insomnia is severe, and their long term use should be avoided as far as possible. The aim should be to give drugs for as short a period as possible, and intermittent use should reduce the risk of dependence. When hypnotics are withdrawn the dose should be reduced gradually.

Benzodiazepines that are promoted for use as hypnotics may be divided into those with short or longer durations of action. Shorter acting benzodiazepines (triazolam, temazepam, and lormetazepam) are indicated for patients for whom residual effects are undesirable, and they are generally preferred when insomnia is not accompanied by daytime anxiety. They are also the most suitable benzodiazepine hypnotics for elderly people, although caution is still required. In some patients the very short acting triazolam may increase wakefulness in the early hours, presumably by a withdrawal mechanism.

Longer acting benzodiazepines such as nitrazepam are indicated when early morning waking is a problem and possibly when an anxiolytic is needed in the daytime and some impairment of psychomotor function is acceptable. In very old people drowsiness, confusion, and unsteadiness can be dangerous, and longer acting benzodiazepines should be avoided.

After the regular use of benzodiazepine hypnotics for only a few weeks rebound insomnia can occur when they are stopped. In 1988 the Committee on Safety of Medicines recommended that benzodiazepine hypnotics should not be taken for more than four weeks and that treatment should be intermittent if possible. 
OTHER DRUGS

The baribiturates can no longer be recommended as hypnotics for the reasons stated above. Chloral hydrate is effective and cheap but may cause side effects, including gastric irritation and rashes. Gastric irritation may be reduced by using one of the derivatives such as dichloralphenazone or triclofos. Although not often the best hypnotic for younger patients, chloral hydrate or one of its derivatives may still be very useful for elderly patients. Chlormethiazole has been recommended for use in the elderly because of its short half life. It often causes nasal and conjunctival irritation when first taken, but this effect may disappear on continuing its use. Chlormethiazole is less safe in overdose than benzodiazepines, and reports of dependence are many. It may be helpful in a few elderly patients where other drugs have failed, and it is sometimes used in alcohol withdrawal although caution is advised.

Drugs of the antihistamine type such as promethazine and trimeprazine have been used as hypnotics, particularly for children, though they have long durations of action and I can see no advantage in their use. Drugs that are not primarily hypnotics may be indicated when insomnia occurs in conjunction with other psychiatric illness. For example, sleep disturbance with depressive illness may respond to the use of sedative antidepressants such as amitriptyline, dothiepin, or mianserin, often given as a single dose at night which thus avoids using hypnotics. Sedative neuroleptics such as thioridazine may be useful for patients with dementia and nocturnal restlessness if given as a single evening dose. Because of potential side effects, however, these drugs should not normally be used as hypnotics unless other indications exist.

A new hypnotic zopiclone (zimovane), a member of the cyclopyrrolone group, has just been released on the British market. It is recommended for short term treatment of insomnia, but its place in management is still not clear.

\section{Conclusions}

After a long period of dominance in the management of anxiety and insomnia benzodiazepines have come under critical review because of the problems of drug dependence although because of their efficacy and safety in overdose they can still be helpful. Their use in longer term treatment is no longer recommended in most cases, although there are probably a few exceptions. In some conditions antidepressants, $\beta$ blockers, and other drugs have a part to play, and it is still too early to define the role of the new anxiolytic, buspirone. More emphasis should now be placed on managing sleep disorders and anxiety without using drugs.

1 Hockings N, Ballinger BR. Hypnotics and anxiolytics. Br Med F 1983;286: 1949-51.

2 Anonymous. Benzodiazepines and dependence: a college statement. Bulletin of the Royal College of Psychiatrists 1988;12:107-9.

A brief statement of college policy.

3 Lader MH. Benzodiazepine dependence. Current Opinion in Psychiatry 1988;1:346-9.

A brief up to date review with annotated references.

4 Catalan J, Gath D, Edmonds G, Ennis J. The effects of non-prescribing of anxiolytics in general practice. Br $\mathcal{F}$ Psychiatry 1984;144:593-602

A trial comparing counselling with anxiolytics in patients with minor affective disorders and somatic complaints.

5 Tyrer P, Murphy S. The place of benzodiazepines in psychiatric practice. Br F Psychiatry 1987;151:719-23.

A useful and balanced account of current psychiatric indications for benzodiazepines.

6 Goa KL, Ward A. Buspirone: a preliminary review of its pharmacological properties and therapeutic efficacy as an anxiolytic. Drugs 1986;32:114-29.

A full review of the properties and uses of this new anxiolytic.

7 Lader $\mathrm{MH}$. Beta-adrenoceptor antagonists in neuropsychiatry: an update. f Clin Psychiatry 1988;49:213-23.

This review includes a discussion of the use of $\beta$ blockers in anxiety disorder, including those characterised by somatic symptoms and performance anxiety.

8 Hollister LE. Pharmacotherapeutic considerations in anxiety disorders. f Clin Psychiatry 1986;47:33-6.

A full review using North American diagnostic classification.

9 Nicholson AN. Hypnotics: their place in therapeutics. Drugs 1988;31:164-76. A useful review of the management of sleep disorder.

\section{MULTICULTURAL MEDICINE}

\section{New transcultural encounters}

"The English have almost everything in common with the Americans except, of course, the language" (Oscar Wilde). The Royal Society of Medicine hosted a two day international conference, entitled "Cancer today," at an elegant hotel in London. Most of the speakers were American professors, sandwiched between some from Britain; there were also a Scandinavian, an Italian, and a New Zealander. The French and Germans either were not invited or stayed away. It is customary that if you have American, British, and sometimes French delegates at a conference it is called an international meeting. On this basis, it is probably just as well that the United Nations is based in New York.

The topic for discussion at the conference was controversial. It was one of the hottest summers that Britain has ever experienced, and there was a lot of hot air both inside and outside the conference hall. One researcher was calling another's work insignificant, only to be accused of making an invalid statement. It is said that you can hear American tourists in London before you can see them. The English also know that the Irish hate only one thing more than a bogeyman and that is another Irishman. At this conference the same could have been said of the American speakers. I suggest that if anyone wants to hold a lively conference he or she should invite some American professors, who will not need loudspeakers, but should also invite some calm British speakers to prevent the temperature of the debate from rising too high.

An interesting cultural conflict occurred at this meeting. After the speeches and question sessions an hour was allocated for discussion among the delegates (about 200) on some of the controversies that exist about cancer. Three people-a hospital consultant, an oncologist, and a general practitioner-were selected to open the debate by speaking for three minutes; the first two were Englishmen, but the general practitioner was a British Asian (the first ever non-European the organisation has selected). Britain has become a multiracial society, as have other countries, without informing the right wing section of its society. The hardest thing in a professional's life is to become accepted by his or her peers. Just before the discussion hour there was a 10 minute break. The consultant discussant approached the British Asian general practitioner and, introducing an English woman doctor, said, "I say, my dear Dr N. I have got a lady doctor who is a general practitioner to speak with me as a third discussant. Perhaps you would not mind not speaking at this AngloAmerican meeting?" The British Asian doctor, who had not slept well the previous night because of worrying about speaking to an all white meeting, had plucked up courage to prepare his three minute speech and, dressed more English than the English, was looking forward to speaking. $\mathrm{He}$ replied, "No sir, I will speak." The lady doctor told the consultant that she would not speak in place of the selected speaker. The consultant was perplexed because the general practitioner had not acted as his houseman would have done. In the middle of this quiet diplomacy the chairman of the conference arrived to organise the sequence for the three discussants. He wrote down the two English names without any reaction but was stunned when he heard the southern Indian name, and his eyes opened wide. The chairman, however, was clever and he divided the name into syllables so as to enable him to pronounce it correctly. It sounded like faya-sri-vasta-wa (first name) Nava-rat-num (surname). The English policy of fair play allowed the British Asian to deliver his speech, which was, in fact, vigorously applauded by the audience.

Incidentally, southern Indians and Sri Lankans are short people with long names. If a surname ends in a vowel the person is a Sinhali (Buddhist) but if it does not the person is a Tamil (Hindu). Asian doctors usually speak good English because English is the second official language in the Commonwealth and medicine is taught mainly in English. Nevertheless, an Oxbridge accent is difficult to understand both for these doctors and for Americans, and the reverse is also true. Speaking slowly, clearly, and loudly (except in the case of Americans) is essential when addressing people from different English speaking countries, especially at an international meeting. If you can not turn the clock back, perhaps there is a way forward. - BASHIR QURESHI, general practitioner, Hounslow, London 